\title{
Photoresponse of Schottky-barrier detector under strong IR laser excitation
}

\author{
S. Ašmontas ${ }^{1}$, D. Seliuta ${ }^{1}$, E. Širmulis ${ }^{2}$ \\ ${ }^{1}$ Semiconductor Physics Institute, A.Goštauto 11, Vilnius 2600, Lithuania, \\ Fax:(3702)627123,e-mail: asmontas@uj.pfi.lt \\ ${ }^{2}$ Institute of Physics, A.Goštauto 12, Vilnius 2600, Lithuania, \\ Fax:(3702) 6170 70,e-mail: sirmulis@ktl.mii.lt
}

\begin{abstract}
Peculiarities of the photovoltaic effect in Ti/n-Si Schottky contact have been studied experimentally under infrared (IR) laser excitation at wavelengths $2.79,3,5,7$ and $10.6 \mu \mathrm{m}$. We demonstrate that strong laser excitation gives rise to the photovoltage even if an incident photon energy is lower than Schottky barrier height. In this case the photovoltage as a function of light intensity follows a power-law dependence with the power greater than unity (2...6). The results are interpreted from the viewpoint of electron emission over the potential barrier due to multiphoton or multistep light absorption at the metal-semiconductor interface.
\end{abstract}

Keywords: Schottky contact, infrared laser, nonlinear photoresponse, multistep light absorption, multiphoton light absorption, surface electronic states

Paper received 05.09.99; revised manuscript received 03.12.99; accepted for publication 14.03.00.

\section{Introduction}

It is known that illumination of metal-semiconductor contact (Schottky barrier junction) gives rise to the photovoltaic effect in a wide spectral region from UV to IR light. The photovoltage mechanism is determined by Schottky barrier height $e \varphi_{B}$, the forbidden energy gap $E_{g}$ and photon energy $h v$. When $h v>E_{g}$, the photocurrent is dominated by electron-hole pair generation followed by pair separation in depletion region of semiconductor (process 1 in Fig. 1). There is also another mechanism, internal photoemission of hot carriers over the Schottky barrier (process 2 in Fig. 1), however, its contribution to the photocurrent in this case is considerably smaller. The latter mechanism prevails when $e \varphi_{B}<h v<E_{g}$.

In this communication we investigate the photovoltage of Schottky barrier junction in IR spectral region when the potential barrier height several times exceeds the incident photon energy. It should be noted that in visible spectral region at metal-vacuum boundary the surface potential barrier for metal electrons is usually $3 \div 5$ times higher than the photon energy. Using different metal targets a third-order [1, 2], fourth-order [3] and fifth-order [4] multiphoton photoemission was obtained. At the metal-electrolyte interface the potential barrier is strongly reduced due to surface electric charges. This allows one to observe a lower-order effect [5]. In metal-semiconductor contact, as far as we know, the nonlinear photoresponse has not been studied by other authors except a theoretical prediction of possibility to observe a two-photon photoeffect in Schottky contact [6]. Two-photon photo-emission from metal to electrolyte using nanosecond excitation of relatively low power density $\left(0.1 \div 3 \mathrm{MW} / \mathrm{cm}^{2}\right)$ [7] suggests that similar effects may be apparently expected in metal-semiconductor structures.

Experimental studies of the Schottky contact photoresponse were performed using nanosecond excitation at wavelengths 10.6 and $2.79 \mu \mathrm{m}$ as well as picosecond excitation at wavelengths 3,5 and $7 \mu \mathrm{m}$. This corresponds to ratio $e \varphi_{B} / h v$ within the range $2 \div 6$. We demonstrate that strong IR laser radiation allows one to observe the photocurrent in metal-semiconductor contact even if the photon energy is much lower than the potential barrier height. We show that $10.6 \mu \mathrm{m}$ radiation leads to multistep electron excitation via real intermediate states, while excitation at wavelengths $2.79,3,5$ and $7 \mu \mathrm{m}$ produces two-photon or three-photon electron photoemission over the Schottky barrier. Our results indicate that dependence of the photocurrent upon laser intensity $P$ is governed by nonlinear relation $R \propto P^{n}(n>1)$ where the power $n$ is determined by the ratio $e \varphi_{B} / h v$. 


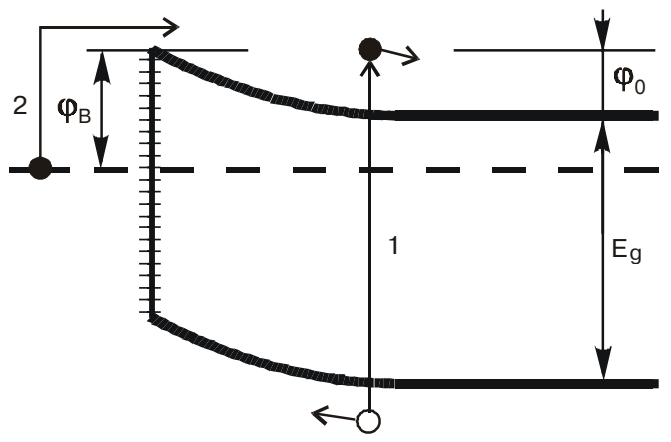

Fig. 1. Schottky contact band diagram: $\varphi_{0}$-diffusion potential, $\varphi_{B}$-Schottky barrier height, $E_{g}$ - forbidden energy gap. The photovoltage mechanisms: 1 - pair generation, 2 - internal photoemission. Dashes represent the interface electronic states.

\section{Experimental details}

The investigated Schottky barrier junctions (Fig. 2) were prepared on n-type monocrystalline silicon substrates (1) of resistivity $1 \mathrm{Ohm} \cdot \mathrm{cm}$. The Schottky contacts of $400 \mu \mathrm{m}$ diameter (4) were formed by deposition of titanium using $d c$ magnetron sputtering followed by thermal annealing at temperature $300^{\circ} \mathrm{C}$ within $30 \mathrm{~min}$. Schottky barrier height $e \varphi_{B}$ determined from the current-voltage (I-V) characteristic, was found to be within the range $0.5 \div 0.6 \mathrm{eV}$. Ideality factor of the $\mathrm{I}-\mathrm{V}$ characteristic was about $1.1 \pm 0.05$.

Experiments at wavelength $10.6 \mu \mathrm{m}$ were carried out by means of passively Q-switched $\mathrm{CO}_{2}$ laser producing $200 \mathrm{~ns}$ pulses at single transverse mode operation [8]. Repetition rate of the output pulses was $30 \mathrm{~Hz}$. Incident power of the laser beam routinely was varied with neutral Teflon filters. For precise measurements a gaseous absorber cell was used. The peak-pulse intensity was measured with germanium photon-drag photodetector. Laser output instability did not exceed $5 \%$ limits.

Experiments at wavelengths $\lambda=2.79 \mu \mathrm{m}$ and $\lambda=3 \div 7 \mu \mathrm{m}$ were performed using nanosecond pulses of erbium (Er: YSGG) laser ( $\tau=60 \mathrm{~ns}$ ) and picosecond pulses of optical
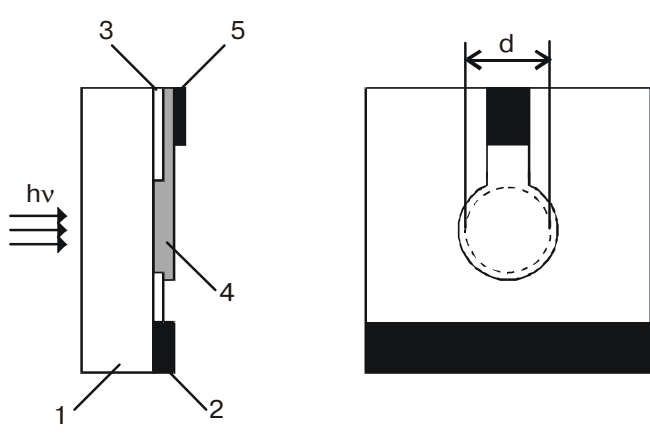

Fig. 2 Schematic view of Ti/n-Si Schottky barrier structure: 1 n-Si substrate, 2 - ohmic contact, 3 - $\mathrm{SiO}_{2}$ layer, 4 - Schottky contact, $5-$ pad for connection. $d=400 \mu \mathrm{m}$.

$S Q O, 3(2), 2000$ parametrical oscillator PG401DFG ( $\tau=15 \mathrm{ps})$, respectively. Both the lasers operated at 1-2 Hz. Energy of the laser pulses was controlled with neutral density filters and measured with a pyroelectric photodetector.

In all the experiments laser beam was perpendicular to the substrate plane. In order to increase excitation intensity the incident beam was focused at a sample surface. Maximum power density was close to $10 \mathrm{MW} / \mathrm{cm}^{2}$. Temporal behaviour of the photosignal and the laser pulse in nanosecond time scale was recorded with digital storage oscilloscope LE'CROY 9360 (sampling rate $5 \cdot 10^{9} \mathrm{~s}^{-1}$ ).

\section{Results and discussion}

Spectral dependence of the Schottky-barrier photoresponse R may be described by the Fowler law [9]:

$R=C \cdot\left[1-\frac{\lambda e \varphi_{b}}{h c}\right]^{2}$,

where $\lambda$ is the excitation wavelength, $C$ is the coefficient of proportionality, $h$ is the Planck constant and $c$ is the speed of light.

Eq. (1) is valid only if the photon energy $h c / \lambda$ is greater than the barrier height $e \varphi_{B}$. It means that the photoresponse rapidly drops down with increase in wavelength and disappears as $\lambda$ reaches the long-wavelength limit $\lambda_{0}$, which is given by $\lambda_{0}=h c / e \varphi_{B}$. For the investigated Ti $/ n$ Si Schottky barrier junctions value of $\lambda_{0}$ was about $2 \div 2.5 \mu \mathrm{m}$. In this work we used excitation at wavelengths $\lambda=10.6 \div 2.79 \mu \mathrm{m}(h v=0.12 \div 0.44 \mathrm{eV})$, i.e. far beyond the long-wavelength limit and observed photosignal for the incident photon energy $2 \div 6$ times smaller than the potential barrier height.

The photovoltage in Til $/ n$-Si Schottky contact had the same polarity as the ordinary internal photoeffect indicating emission of electrons from metal to semiconductor over the potential barrier. This is reverse situation compared to the hot-electron photovoltaic effect in $p-n$ or $l-h$ semiconductor junctions [10, 11].

Photovoltage $U_{p}$ as a function of $\mathrm{CO}_{2}$ laser intensity is depicted in Fig. 3. Dependence $U_{p}(P)$ demonstrates steep superlinear growth followed by decrease in slope and saturation at higher light intensities. The photosignal saturation is observed because maximum value of the photovoltage is limited by the junction diffusion potential $\varphi_{0}$. It is seen that the photosignal is strongly affected by internal electric field which depends on external bias voltage. Bias dependence of the saturated value of $U_{p}$ we attribute to $\varphi_{0}$ variation with external voltage. (Following investigations were made at zero bias).

More precise measurements of the superlinear part of characteristic $U_{p}(P)$ (using gas absorber cell) show that the plot in log-log scale consists of two linear regions with two different slopes (Fig. 4). It means that relation between $U_{p}$ and $P$ is governed by power-law dependence $U_{p} \propto P^{n}$ with different values of $n$.

Oscilloscope traces of the photovoltage signal and $\mathrm{CO}_{2}$ laser pulse are shown in Fig. 5. Curves 2-4 illustrate 
S. Ašmontas et al.: Photoresponse of Schottky-barrier detector under strong ...

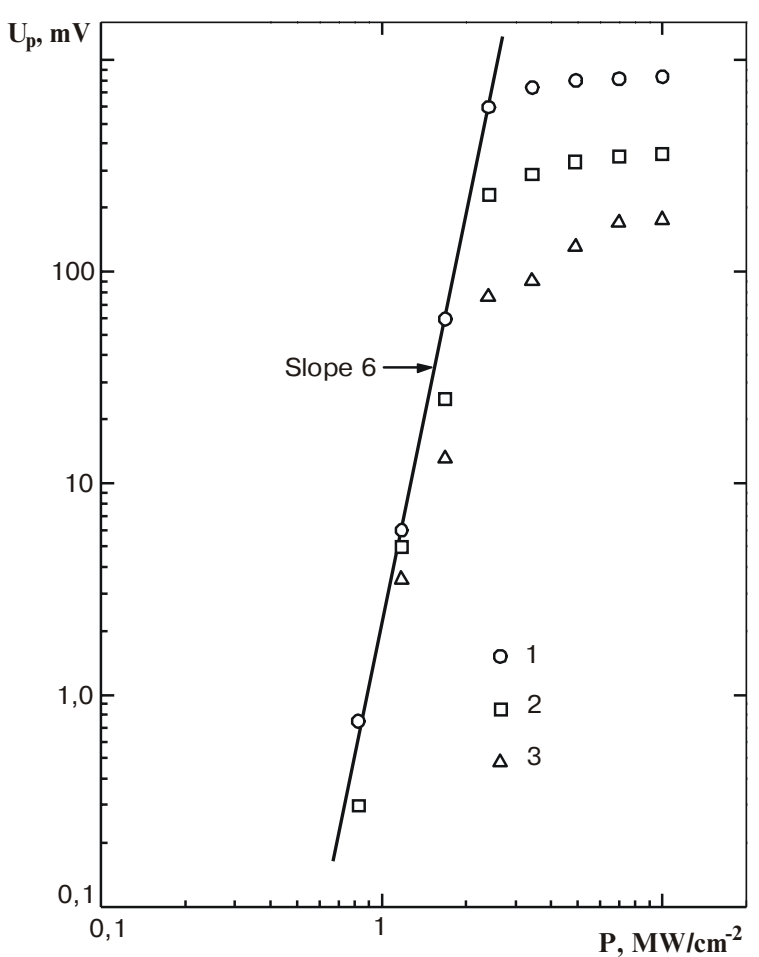

Fig. 3 The photovoltage versus $\mathrm{CO}_{2}$ laser intensity $(\lambda=10.6 \mu \mathrm{m})$ for different bias values: $1--0.5 \mathrm{~V}, 2-0 \mathrm{~V}, 3-+0.2 \mathrm{~V}$.

the photovoltage signal corresponding to different laser intensity levels $\left(P_{2}<P_{3}<P_{4}\right)$ and curve 1 represents the laser pulse trace. At low intensity levels the photosignal is narrowed with respect to the laser pulse (trace 2). This is related with the nonlinear relation between the photovoltage and laser intensity. We found that the narrow trace corresponds to the left part of the plot in Fig. 4. In this region of light intensities value of the power $n$ is governed by relation:

$n=\left[\frac{e \varphi_{B}}{h v}+1\right]$

where angle brackets mean an integral part of the expression.

At higher values of $P$ the photosignal pulse width starts to increase (Fig. 5, traces 3 and 4). At the same time tendency of increase in slope is observed (Fig. 4), indicating an increased nonlinearity of the photovoltaic effect.

The discussed results were obtained at room temperature. At liquid nitrogen temperature we observed nearly twofold increase in magnitude of the signal, however, any variation in slope of characteristic $U_{p}(P)$ did not occur.

It should be noted that in the case of $\mathrm{CO}_{2}$ laser excitation $(\lambda=10.6 \mu \mathrm{m}, h v=0.117 \mathrm{eV})$ value of the power $n$ obtained from Eq.(2) is equal to 6. It means that total energy of six quanta is needed for electron to overcome the potential barrier of the Schottky contact. However, experimental data on four-photon and five-photon elec-

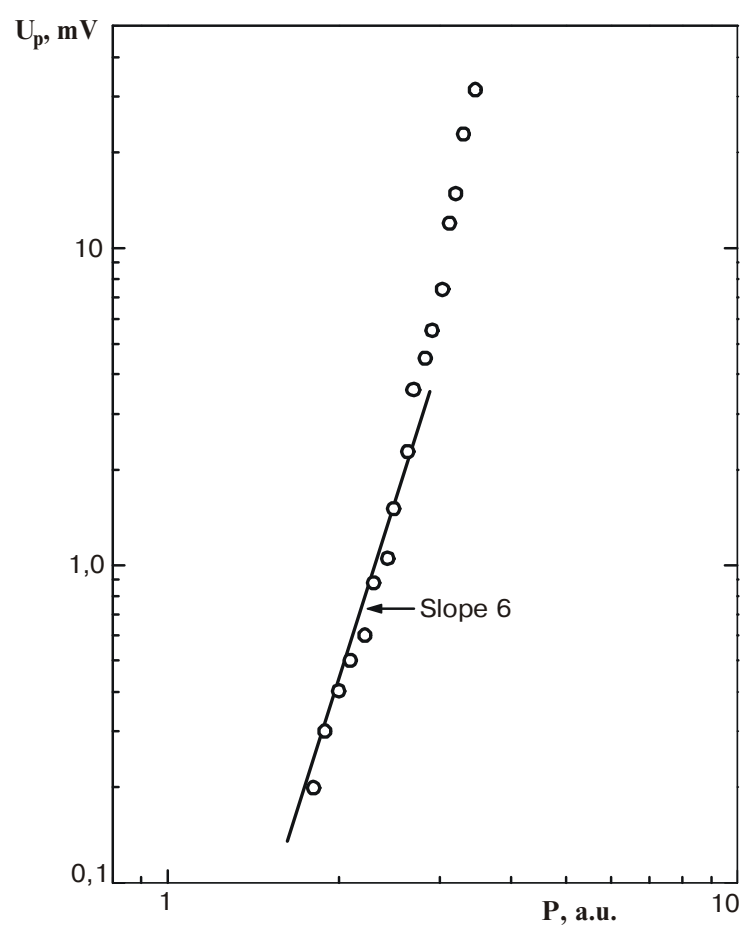

Fig. 4 Fragment of the plot in Fig. 3 at zero bias voltage.

tron photoemission at metal-vacuum boundary $[3,4]$ as well as theoretical estimation of the multiphoton excitation rate [12] suggest that probability of pure six-photon effect is extremely small. Therefore, in the investigated range of light intensities the photovoltage value should be by many orders of magnitude less than that observed in our experiments. Yet it is known that metal-semiconductor interface contains a considerable number of electron energy states distributed within the forbidden en-

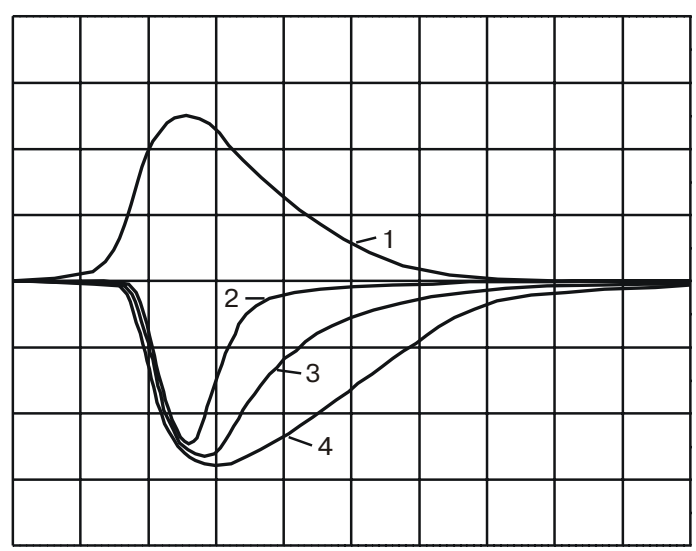

Fig. $5 \mathrm{CO}_{2}$ laser pulse trace (1) and the photovoltage pulse traces $(2,3,4)$ for different laser intensities $P: P_{2}<P_{3}<P_{4}$. Time scale $100 \mathrm{~ns} / \mathrm{div}$. 


\section{S. Ašmontas et al.: Photoresponse of Schottky-barrier detector under strong ...}

ergy gap. Therefore, there appears an additional channel of multistep electron excitation via real intermediate states $[13,14]$.

Let us now consider a simplest (second-order) nonlinear process of electron-hole pair generation in semiconductor. In general case, the generation rate is given by [15]:

$$
g=\frac{N \sigma_{1} \sigma_{2} P^{2}}{\frac{1}{\tau}+\left(\sigma_{1}+\sigma_{2}\right) P}+\alpha_{2} P^{2}
$$

where the first term represents the two-step generation rate and the second term stands for the two-photon generation rate; $N$ is the number of intermediate energy states per unit volume, $\tau$ is the intermediate state life time, $\sigma_{1}$ and $\sigma_{2}$ are the absorption cross-sections and $\alpha_{2}$ is the twophoton absorption coefficient. It is assumed here that electron transition probabilities (valence band - intermediate state and intermediate state - conduction band) are proportional to light intensity with coefficients of proportionality $\sigma_{1}$ and $\sigma_{2}$, respectively.

It follows from Eq.(3) that at low laser intensities when $\tau^{-1}>>\left(\sigma_{1}+\sigma_{2}\right) P$ the two-step as well as the two-photon generation rate is proportional to $P^{2}$. Therefore, the second-power law dependence of the photosignal on light intensity does not provide identification of the physical process. Furthermore, one can show that in the case of $n$-th order photoeffect the multistep as well as the multiphoton excitation rate (at low intensity levels) is proportional to $P^{n}$.

Since the electron life time in a real intermediate state strongly exceeds that in a virtual state probability of the multistep process is much greater than that of the multiphoton effect [16] and the difference rapidly grows up (following the power-law dependence) as the coefficient of nonlinearity $n$ is increased. Therefore, magnitude of the photocurrent originating from six-step light absorption should be comparable with our experimental data.

We found experimentally that thermal treatment of samples using powerful laser radiation is necessary in order to obtain the nonlinear photosignal. We assume that initial number of surface energy states is not sufficient for the multistep photoeffect to be measurable. Therefore, heating of metal-semiconductor interface with $\mathrm{CO}_{2}$ laser pulses at high power density (about $10 \mathrm{MW} / \mathrm{cm}^{2}$ ) generates additional electronic states and considerably increases the multistep excitation rate. Energy distribution of the interface states is not known, however, we suppose that they are distributed quasicontinuously [17] and every virtual state is supported by sufficient number of interface states. From this we conclude that in case of $\lambda=10.6 \mu \mathrm{m}$ the six-step electron photoemission is responsible for the observed photovoltage in Ti/n-Si Schottky contact.

In addition, it has to be noted that the increase in slope of the plot $U_{p}(P)$ at high laser intensities (Fig. 4) does not mean an increased number of photons participating in a process of nonlinear light absorption. It implies rather an excessive heating of metal surface followed by the ther- mal generation of electron-hole pairs. It is known that the thermal generation rate is characterised by strong (exponential) dependence on the surface temperature. This is in some agreement with nonlinear electron photoemission data obtained for the metal-vacuum interface [1, 2]. Presence of the thermal component of the photovoltage at high excitation intensities manifests itself in prolonged shape of the photosignal traces (Fig. 5).

Using laser light sources operating at shorter wavelengths we were able to explore lower-order nonlinear photoeffects. For example, in the case of erbium laser excitation $(\lambda=2.79 \mu \mathrm{m}, h v=0.44 \mathrm{eV})$, relation between the photon energy and the barrier height is given by inequality $h v<e \varphi_{B}<2 h v$, therefore, in accordance with Eq.(2) value of the power $n$ should equal 2. Experimental results also indicate the second-order effect (Fig. 6). The emitted charge was calculated by integrating the photocurrent pulse. This allows one to avoid the photosignal saturation at high laser intensities caused by screening of internal electric field of the junction.

Similar behaviour of the photovoltage was observed using pulses of the optical parametrical oscillator at wavelengths 3, 5 and $7 \mu \mathrm{m}$. Relations between the Schottky barrier height and the photon energy now are as follows:

$$
\left\{\begin{array}{l}
h v<e \varphi_{B}<2 h v, \text { for } \lambda=3 \mu m, \\
2 h v<e \varphi_{B}<3 h v, \text { for } \lambda=5 \mu m \text { and } \lambda=7 \mu m .
\end{array}\right.
$$

Consequently, in the first case the second-order process is expected to prevail, while in the second case - the third-order one. Experimental data confirmed these predictions (Fig. 7): for $\lambda=3 \mu \mathrm{m}$ the photovoltage follows a square-law dependence on intensity; for $\lambda=5 \mu \mathrm{m}$ and $\lambda=7 \mu \mathrm{m}$ the dependence is close to a cubic-law.

Using nanosecond pulses produced by erbium laser at $\lambda=2.79 \mu \mathrm{m}$ we found that at low light intensities the photovoltage pulse again is narrowed with respect to the laser pulse indicating the absence of any thermal effect. In the case of excitation at wavelengths 3,5 and $7 \mu \mathrm{m}$ we suppose that picosecond light pulses in the investigated

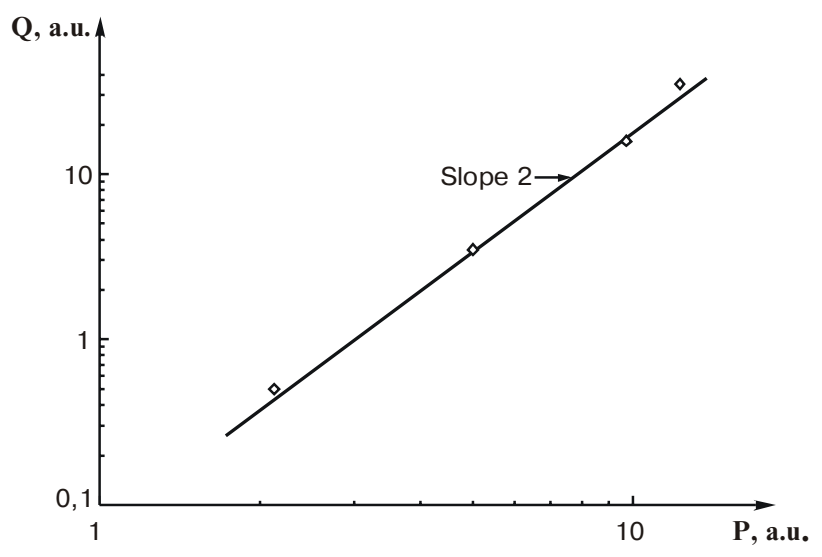

Fig. 6 The emitted charge versus erbium laser intensity $(\lambda=$ $2.79 \mu \mathrm{m}) . T=300 \mathrm{~K}$ 

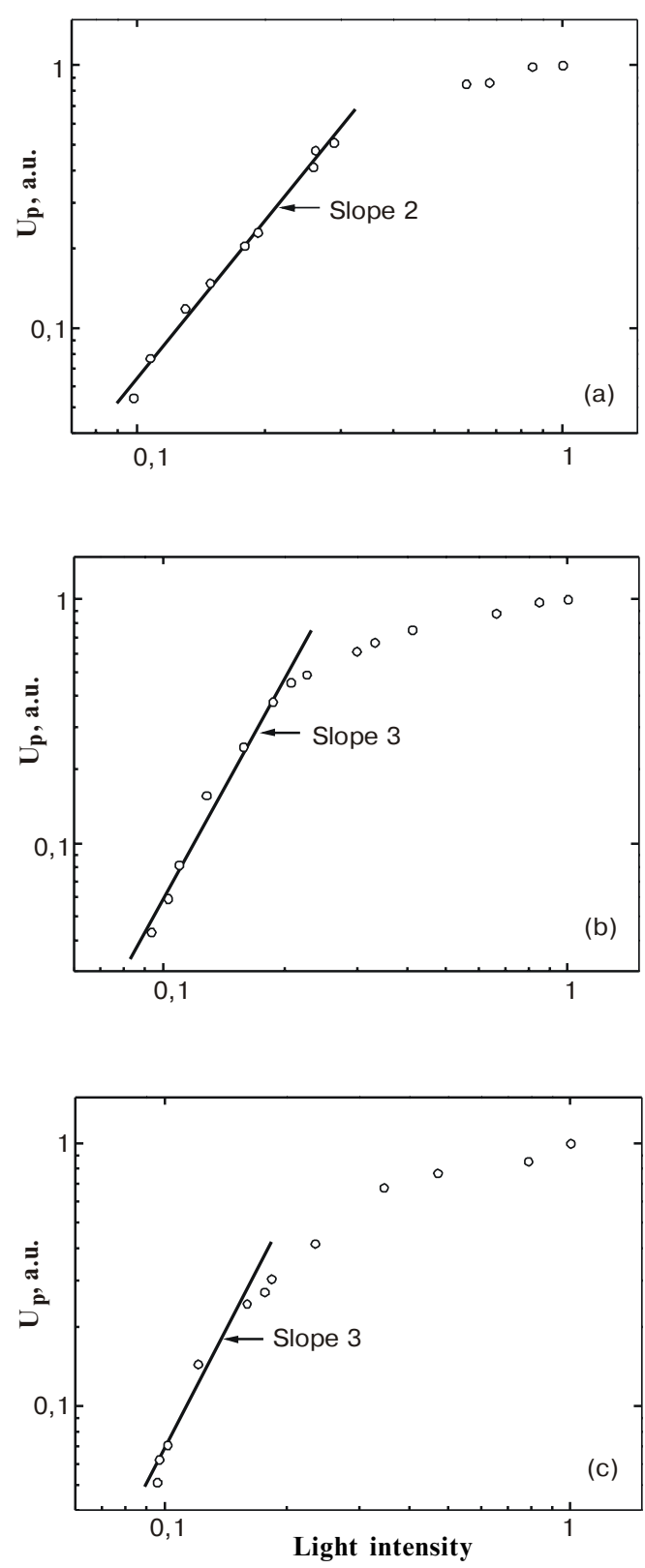

Fig. 7 The photovoltage versus light intensity (in arbitrary units) at different excitation wavelengths $\lambda$ : (a) $-3 \mu \mathrm{m}$, (b) $-5 \mu \mathrm{m}$, (c) $7 \mu \mathrm{m} . T=300 \mathrm{~K}$

intensity range do not cause any significant heating of metal surface [18] as well. So the problem is: which process - multistep or multiphoton - is responsible for the observed photovoltage. To answer this question one should note that, firstly, any thermal treatment of samples is not needed in this spectral range what speaks in favour of the multiphoton effect. In addition, magnitude of the twophoton and the three-photon absorption is by many orders of magnitude greater than the six-photon effect and thus should be comparable with our experimental results. Therefore, we suppose that the multi-photon electron photoemission is prevailing at excitation wavelengths within the range $2.79 \div 7 \mu \mathrm{m}$.

\section{Conclusions}

We have shown that the photoresponse in Ti/n-Si Schottky barrier can be observed even when the photon energy is much lower than the potential barrier height. We found that dependence of the photoresponse $R$ upon laser intensity $P$ is governed by relation $R \propto P^{n}$, where value of the power $n$ corresponds to the number of photons participating in the photoemission of electron. We suggest that laser radiation at wavelength $10.6 \mu \mathrm{m}$ gives rise to multistep electron excitation via real intermediate states, while excitation at shorter wavelengths produces two-photon or three-photon electron emission over the Schottky barrier.

\section{Acknowledgements}

We would like to thank the EKSPLA Co., Ltd. in Vilnius for providing the optical parametrical generator PG401DFG and Er:YSGG laser used in this work. We are grateful for support and encouragement of Dr. P.Balkevicius and Dr. A.Michailovas and the kind technical assistance of A. Rinkevicius and E. Babrauskas during the experiments. This work was partly supported by Lithuanian State and Education Fund within the framework of «Jutikliai» Programme.

\section{References}

1. G. Farkas, I. Kertesz, Z. Naray, P. Varga, On the laser-induced non-linear photoelectric effects in metals // Phys.Lett., 25A, pp. 572-573 (1967).

2. E.M. Logothetis, P.L. Hartman, Laser-induced electron emission from solids: many-photon photoelectric effects and thermionic emission // Phys. Rev., 184, pp. 460-474 (1969).

3. J.H. Bechtel, W.L. Smith, N. Bloembergen, Four-photon photoemission from tungsten // Opt. Commun., 13, pp. 56-59 (1975).

4. G. Farkas, Z.Gy. Horvath, Multiphoton electron emission processes induced by different kinds of ultrashort laser pulses // Opt. Commun., 12, pp. 392-395 (1974).

5. S.D. Babenko, V.A. Benderskii, A.G. Krivenko, T.S. Rudenko, Polarisation properties of one- and two-photon photoeffect at metal-electrolyte interface // Fiz. Tverd. Tela, 16, pp. 13371341 (1974).

6. V. K. Kanonenko, Two-photon effect in metal-semiconductor structures // Zh. Tekh. Fiz, 49, pp. 2262-2265, (1979) (in Russian).

7. S.D. Babenko, V.A. Benderskii, T.S. Rudenko, Observation of high-probability two-photon photoemission from metals to electrolyte solutions // Letters to Zhurn. Eksp. Teor. Fiz., 17, pp. 71-74 (1973).

8. A. Merkys, S. Stonys, E. Širmulis, A simple current pulsed low-pressure $\mathrm{CO}_{2}$ laser with passive Q-switching // Optical and Quantum Electron., 20, pp. 373-376 (1988).

9. H. Elab and W. Kosonocky, Theory and measurements of photoresponse for thin films $\mathrm{Pd}_{2} \mathrm{Si}$ and $\mathrm{PtSi}$ infrared Schottkybarrier detectors with optical cavity // RCA Review, 43, pp. 569-572 (1982).

10. M. Umeno, Y. Sugito, T. Jimbo, H. Hattori, Y. Amemiya, Hot photo-carrier and hot electron effects in junctions // Solid. St. Electron., 21, pp. 191-195 (1978).

11. S. Ašmontas and E. Širmulis, Heating of holes in silicon under $\mathrm{CO}_{2}$-laser excitation // Phys. Tekh. Poluprovodn., 20, pp. 2212-2215 (1984) (in Russian). 


\section{S. Ašmontas et al.: Photoresponse of Schottky-barrier detector under strong ...}

12. F.V. Bunkin and A.M. Prokhorov, Some peculiarities of laser-mater interaction using short excitation pulses // Zhurn. Eksp. Teor. Fiz., 52, pp. 1610-1615 (1967) (in Russian).

13. A. Z. Grasiuk, I. G. Zubarev, A.B. Mironov, I. A. Poluektov, The features of the two-photon interband absorption spectra of the laser radiation in GaAs // Phys. Tekh. Poluprovod., 10, pp.262-270 (1976) (in Russian).

14. R. Baltramiejunas, V. Gavriusin, J. Vaitkus, The effect of impurities on the two-photon absorption in CdS monocrystals // Phys. Tverd. Tela, 18, pp.1150-1153 (1975) (in Russian).

15. D.P. Dvornikov, E. L. Ivchenko, V. V. Pershin, I. D Jarosheckii, Effect of the transitions via deep impurity centers to the process of the nonlinear light absorption in semiconductors // Phys. Tekh. Poluprovod., 10, pp.2316-2319, 1976 (in Russian).

16. I.V. Lebedev, Multiphoton ionisation of semiconductors with impurity states // Optika i Spektrosk., 33, pp. 1190-1191 (1972) (in Russian).

17. V.I. Strikha, E.V.Buzaneva, I.A.Radzievskii, Semiconductor devices based on Schottky contact, Sov. Radio, Moscow (1974).

18. S.I.Anisimov, N.A.Inogamov, Y.V.Petrov, Intensity dependence of a laser initiated emission current from a metal surface // Phys. Lett., 55A, pp. 449-450 (1976). 